
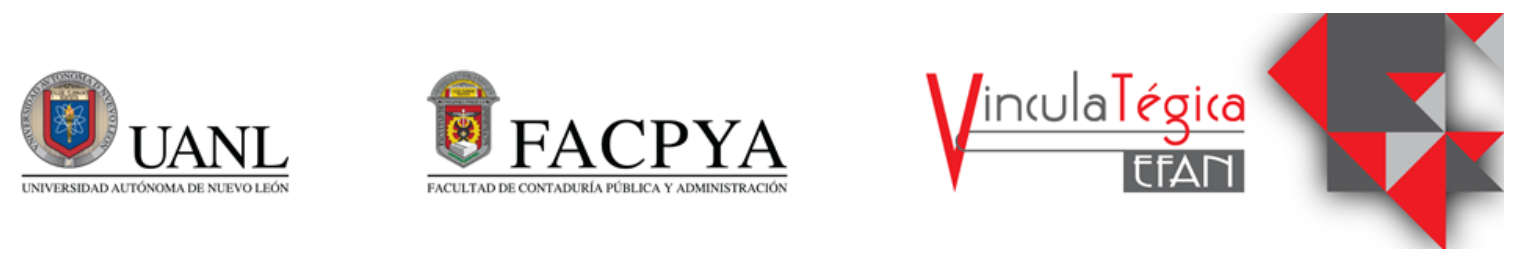

\title{
Salud ocupacional y trabajo remoto durante la pandemia: riesgos y recomendaciones
}

\author{
Cynthia M. Tomas Montaudon ${ }^{1}$, Ingrid N. Pinto López ${ }^{2}$ y Anna Amsler ${ }^{3}$ \\ ${ }^{1}$ UPAEP, cynthiamaria.montaudon@ upaep.mx, 21 Sur 1103 Barrio de Santiago, +52 2222299400 \\ ${ }^{2}$ UPAEP, ingrid.pinto@upaep.mx, 21 Sur 1103 Barrio de Santiago, +52 2222299400 \\ ${ }^{3}$ Consultor independiente, annaamsler95@gmail.com
}

\begin{abstract}
Información del artículo revisado por pares
Fecha de aceptación: junio-2021

Fecha de publicación en línea: diciembre-2021

DOI: https://doi.org/10.29105/vtga7.2-54
\end{abstract}

\section{Resumen}

La pandemia de COVID-19 trajo consigo un incremento sustancial del trabajo remoto que inició como una respuesta de emergencia, pero que se mantuvo durante más de un año en ciertos sectores y funciones. Hasta antes de la pandemia, se habían analizado los beneficios del trabajo remoto en cuanto a la salud mental, el bienestar general y el incremento en la productividad laboral, bajo condiciones donde el trabajador decidía cómo y dónde quería trabajar. La modificación forzada a la rutina laboral y a las condiciones generales del empleo resultaron en una serie de afectaciones negativas tanto en la salud como en el bienestar general de los trabajadores remotos. Este artículo presenta una recopilación exhaustiva de los riesgos y problemas en la salud ocupacional de los empleados remotos, así como recomendaciones para reducirlos y evitarlos.

Palabras clave: Trabajo remoto, salud ocupacional, bienestar, recomendaciones

Códigos JEL: J4, J41, J81, J2, J22

\begin{abstract}
The COVID-19 pandemic brought a substantial increase in remote work, which began as an emergency response but continued for more than a year in certain sectors and functions. Until before the pandemic, the benefits of remote work had been analyzed in terms of mental health, general well-being and increased labor productivity, under conditions where the worker decided how and where to work. The forced transformation of the work routine and general employment conditions resulted in a series of negative effects on both the health and general well-being of remote workers. This article presents a comprehensive compilation of the occupational health risks and problems of remote workers, as well as recommendations for reducing and avoiding them
\end{abstract}

Key words: Remote work, occupational health, wellness, recommendations

JEL Codes: J4, J41, J81, J2, J22 


\section{INTRODUCCIÓN}

Durante la Revolución Industrial, las condiciones de trabajo generaban riesgos importantes de accidentes y problemas de salud, particularmente debido a humos y productos nocivos a los cuales estaban expuestos los trabajadores de todas las edades, incluso niños, resultando en enfermedades severas y una alta tasa de mortalidad (Cameron, 2021). Desde entonces, se ha avanzado de manera importante en el desarrollo de medidas diversas como mejorar las condiciones de iluminación y ventilación, proveer equipo de protección, incorporación de los principios de ergonomía, entre otros, para reducir los problemas de salud y los accidentes y mejorar las condiciones de vida laboral.

La salud ocupacional comenzó a tomar mayor importancia a partir de 1970 con el desarrollo de diversas normativas internacionales para garantizar condiciones de trabajo sanas y seguras para todos los trabajadores, independientemente del sector en el que se desarrollaban (EHS Insight Resources, 2019). Inicialmente, el enfoque era el análisis y mejoramiento de las condiciones laborales de quienes realizaban trabajos manuales rutinarios, como es el caso de trabajadores de manufacturas, donde con frecuencia se contrataba a jóvenes sin experiencia y el lugar de trabajo estaba sometido a riesgos constantes que causaban accidentes y otros problemas de salud.

En 2020, la pandemia de COVID-19 trajo consigo la reconsideración sobre la salud ocupacional debido a las nuevas designaciones de trabajadores y a las condiciones de distanciamiento impuestas.

El Foro Económico Mundial, (2020) estableció tres categorías de trabajadores durante la crisis sanitaria:

- Empleados esenciales: personas cuyos empleos se relacionan con mantener la seguridad, la salud y el bienestar. Esta clasificación incluye trabajadores de primera línea (bomberos, policías, autoridades del sistema penitenciario, trabajadores agrícolas y de la industria alimentaria, industria manufacturera esencial, tiendas de alimentos, servicios de comida, transporte público, sector educativo, transporte y logística, finanzas, construcción de vivienda, energía, derecho, tecnologías de la información, comunicaciones, medios de comunicación, entre otros).

- Empleados remotos: personas que trabajan fuera de las instalaciones del empleador. Trabajan de manera remota y pueden conservar su empleo trasladándose al trabajo desde casa donde cuentan con las condiciones básicas de acceso a internet y otras tecnologías.

- Empleados no esenciales: personas cuyos empleos han sido suspendidos o limitados a causa de las restricciones sanitarias. Entre los sectores más afectados se encuentra el sector turístico y restaurantero, tiendas de ropa y accesorios, guarderías, gimnasios, eventos deportivos y empresas de entretenimiento, entre otros.

Esta denominación ha hecho necesaria una reconsideración sobre la salud ocupacional de los trabajadores en condiciones distintas a las previamente establecidas.

Este estudio presenta una revisión de la literatura existente sobre los riesgos y efectos del trabajo remoto en la salud y bienestar de los trabajadores, así como una serie de recomendaciones para evitar dichos riesgos o minimizar los problemas de salud asociados a esta forma de trabajo deslocalizada.

El objetivo principal es identificar las investigaciones elaboradas con respecto a la salud ocupacional en términos del teletrabajo con el fin de coadyuvar al desarrollo de marcos teóricos para estudios futuros o investigaciones empíricas sobre padecimientos específicos asociados al 
trabajo remoto durante y después de la pandemia.

\section{MARCO TEÓRICO}

\subsection{Salud ocupacional}

La salud ocupacional ha sido una preocupación constante de empresas y gobiernos. La Organización Mundial de la Salud (2021), ha establecido que la salud de los trabajadores es esencial, por lo que ha generado parámetros que permiten mejorar las condiciones en el contexto laboral. Este organismo ha establecido que la salud ocupacional se centra en todos los aspectos de la salud y la seguridad en el lugar de trabajo y tiene un fuerte enfoque en la prevención de riesgos.

La salud de los trabajadores tiene varios determinantes, incluidos los factores de riesgo en el lugar de trabajo que conducen a enfermedades, accidentes, lesiones musculoesqueléticas, enfermedades respiratorias, hipoacusia, enfermedades circulatorias, trastornos relacionados con el estrés y enfermedades transmisibles, entre otros (Ibidem).

Las políticas tendientes a mejorar la salud ocupacional tienen como objetivo la adaptación del entorno laboral a los trabajadores para promover el más alto grado de bienestar físico, mental y social. Son importantes para el trabajador, pero también para la organización en su conjunto ya que una fuerza laboral sana y motivada tiende a generar mejores resultados (Tadesse y Admassu, 2006).

En este sentido, desde 1995 la Organización Mundial de la Salud previó que uno de los retos más importantes en términos de salud ocupacional en el futuro serían los problemas vinculados al uso creciente de las Tecnologías de la Información y la Comunicación (Ibídem).

\subsection{Trabajo remoto}

El teletrabajo, o trabajo remoto, es una modalidad de empleo que considera al hogar, una cafetería, espacios de coworking, el transporte y otros lugares públicos y privados como una extensión del trabajo. Bajo este esquema se elimina la necesidad de un espacio fijo para realizar la actividad laboral y se utilizan diversas tecnologías para el desarrollo de las funciones (Foro Económico Mundial, 2020). Nuevas aplicaciones digitales permiten mejorar los flujos de trabajo, promover nuevas formas de almacenar y compartir información y proveer la posibilidad de movilidad y conectividad remota. Estas innovaciones han transformado el trabajo en algo más fluido, informal, flexible y móvil (Organización Internacional del Trabajo, 2017). Así, el trabajo remoto se ha convertido en una estrategia sustentable que permite a ciertos sectores y áreas en las empresas, operar aún en condiciones de distanciamiento social.

El potencial del trabajo remoto está sujeto a las características del trabajo a desempeñar y a la infraestructura disponible (Berg, Bonnet y Soares, 2020). El teletrabajo puede no ser un ajuste ideal para todos los trabajadores, ya que, en situaciones como la pandemia, hace coincidir las responsabilidades del hogar con las del trabajo (Organización Internacional del Trabajo, 2020; Allen, Cho y Meier, 2014).

Las condiciones impuestas al modificarse las características del empleo han obligado al desarrollo de políticas que permitan aplicar los derechos y las obligaciones de salud ocupacional incluyendo las precauciones necesarias tanto para el empleado como el empleador en el teletrabajo y los modelos de trabajo híbrido (Chandler, 2020).

\subsection{Condiciones de trabajo durante la pandemia de COVID-19}

Cuando el trabajo remoto se impuso de manera emergente, había una preocupación en las empresas de que los empleados utilizaran la mayor parte de su tiempo para atender problemas personales y familiares en lugar del trabajo. Actualmente, la preocupación se ha 
movido al análisis de la falta de límites a los horarios de trabajo remoto. Se han realizado estudios que demuestran que las personas que trabajan desde casa tienden a trabajar más horas, debido al limitado control del tiempo que trabajan (Organización Internacional del Trabajo, 2017), incluso trabajan durante los fines de semana (Potratz, 2020).

El cambio al trabajo remoto durante la crisis sanitaria se llevó a cabo de manera abrupta. No hubo tiempo suficiente para preparar los espacios del hogar para desarrollar trabajo de oficina que, por lo general, conlleva estar sentado frente a una computadora más de ocho horas. El trabajo pasó de realizarse en un espacio diseñado para ello a llevarse a cabo en la mesa de la cocina o del comedor, el sillón de la sala o en la recámara. En algunos casos, las personas contaban con un escritorio y una silla, pero quizás sin la capacidad de ajuste necesaria que proporciona un entorno de trabajo adecuado (Organización Internacional del Trabajo, 2020).

Este contexto, de la mano con otros factores, puede afectar la salud y el bienestar laboral de manera negativa, contribuyendo también a una disminución en la productividad y el desempeño de los trabajadores. Los principales riesgos ocupacionales del trabajo remoto son de tipo ergonómicos, psicosociales y aquellos derivados de la dificultad de separar la vida familiar de la laboral.

\section{MÉTODO}

Este estudio fue realizado a través de un análisis de la literatura y la compilación de los distintos problemas de salud y riesgos identificados. Se desarrolló un análisis bibliométrico en la red de las ciencias (Web of Science, WoS, 2021) utilizando como palabras clave "trabajo remoto" y "salud ocupacional", así como diversos sinónimos en su versión en inglés. Dicho análisis arrojó un total de 138 publicaciones, que iniciaron en el año 2012, mostrando variabilidad en distintos años y alcanzando su punto más alto en 2020 . La tendencia parece indicar que el número de publicaciones continuará aumentando, ya que, para mayo de 2021, se tenían 11 publicaciones registradas.

Tabla 1 Total de materiales publicados que analizan el trabajo remoto y la salud ocupacional

\begin{tabular}{cc}
\hline Año & $\begin{array}{c}\text { Total de } \\
\text { Publicaciones }\end{array}$ \\
\hline 2012 & 1 \\
2013 & 6 \\
2014 & 8 \\
2015 & 15 \\
2016 & 7 \\
2017 & 8 \\
2018 & 13 \\
2019 & 10 \\
2020 & 18 \\
2021 & 11 \\
\hline
\end{tabular}

Fuente: Red de las Ciencias (WoS, 2021), búsqueda realizada en mayo de 2021.

Es importante destacar que la gran mayoría de las publicaciones, es decir, 121, corresponden a la categoría de artículos, 16 a revisiones a diversas publicaciones, 8 participaciones en congresos, una nota editorial y 4 artículos más de acceso temprano en proceso de publicación.

Las publicaciones fueron desarrolladas principalmente en Australia (40\%), Estados Unidos (21\%), Canadá (17\%), Reino Unido, (9\%) y, en menor medida, en otros países de Europa, Asia, Sudamérica y África (13\%).

Un análisis detallado de las publicaciones permitió identificar que sólo $39.8 \%$ de estas abordan temas vinculados específicamente a las condiciones de salud ocupacional en el trabajo remoto. Además, solo el $21 \%$ se centran en el contexto de la pandemia de COVID-19, lo cual permite observar que existe un área de análisis poco explotada, debido a las condiciones sin precedente en el mundo laboral.

Adicional a la búsqueda de publicaciones en la Red de las Ciencias, se identificaron publicaciones de diversos organismos y empresas consultoras y proveedoras de servicios para el trabajo remoto que habían desarrollado estudios sobre los efectos del trabajo a distancia durante la pandemia. 
A partir de la información obtenida se establecieron categorías de riesgos y padecimientos, así como recomendaciones emitidas por diversas fuentes.

\section{RESULTADOS}

\subsection{Principales problemas de salud asociados al teletrabajo}

Numerosos estudios han examinado los aspectos psicológicos y fisiológicos del trabajo remoto, obteniendo resultados diversos (Maruyama, Hopkinson y James, 2009). Por un lado, se habla de mayores beneficios para los empleados en términos de libertad para elegir dónde y cuándo trabajar y cómo organizarse; sin embargo también se ha evaluado la problemática de la dificultad de desconexión y otros factores que inciden de manera negativa en el bienestar de los teletrabajadores.

Uno de los principales problemas que el trabajo remoto ha creado en torno a la salud ocupacional es que el empleador tiene una capacidad limitada para monitorear las condiciones de trabajo desde casa y las horas trabajadas, así como las cargas de trabajo de sus empleados (The Center for Occupational Safety, 2020). Si a esto se le suma que millones de personas han estado usando las computadoras mucho más tiempo que antes para estudiar y trabajar, y han tenido que estar en condiciones de confinamiento dentro de sus hogares, la problemática se agrava.

Los problemas de salud identificados en la literatura se clasificaron en problemas de salud física y salud mental. Los riesgos más importantes se incluyeron como una categoría distinta.

\subsubsection{Problemas de salud física}

\section{Tech neck}

Factores como la posición encorvada al trabajar en una computadora portátil, el estar en una misma posición por periodos prolongados, la reducción de la actividad física por el confinamiento y la falta de mobiliario ergonómico han incidido en el aumento de los casos de tensión en cuello y espalda (Upham, 2021). El llamado tech-neck, o cuello tecnológico, es un nuevo término que se utiliza para describir un problema antiguo: dolor de cuello causado por tensión repetitiva y lesiones en los músculos y otras estructuras tisulares de la columna cervical (Miller, 2020).

Es uno de los riesgos de salud más predominantes del trabajo remoto, resultado del estrés causado en los músculos del cuello, espalda y hombros por la postura adoptada frente a la computadora (UNUM, 2020). Trabajar en computadoras portátiles agrava la situación, ya que mientras más se inclina la cabeza por muchas horas, más severas son las lesiones, que pueden resultar en discos dañados y nervios presionados (Miller, 2020).

\section{Dolor en la espalda baja}

Diversos estudios han sugerido que el dolor musculoesquelético más común durante la pandemia ha sido el dolor de la espalda baja, producido por condiciones de trabajo poco óptimas, como el caso del uso de mobiliario que no es ergonómico. De acuerdo con Moretti, Menna, Bulichino, Aulicino, Paoletta, Liguori y Iolascon (2020), este hallazgo no es sorprendente, considerando que el dolor de columna es uno de los problemas de salud más frecuentes en la población en edad laboral a nivel mundial y existe evidencia que sustenta que un trabajo sedentario sostenido en una postura forzada es un factor de riesgo adicional de dolor lumbar. De hecho, los dolores y problemas de espalda han sido reportados como una de las principales afecciones de los trabajadores remotos (Di Donato, Iles, Buchbinder, Xia, y Collie, 2021)

Se ha argumentado que el riesgo de dolor lumbar parece aumentar cuando las personas permanecen sentadas más de 7 horas al día (Moretti et al., 2020), aunque la incidencia de este padecimiento también está asociado con factores como la edad, el índice de masa corporal, la posición del cuerpo al sentarse, el tipo de respaldo y la distancia corporal con respecto a la pantalla de la computadora. 


\section{Dolor de articulaciones, síndrome del túnel carpiano y tendinitis}

Empleados que sufren lesiones por estrés físico repetitivo al trabajar en casa se ha convertido en un problema creciente que más empresas deberán abordar (Porter, 2021). Como se mencionaba anteriormente, la falta de un espacio adecuado y habilitado para el trabajo remoto y el uso de dispositivos digitales como tabletas, computadoras portátiles y teléfonos celulares para trabajar ha provocado que numerosos teletrabajadores experimenten dolor en las articulaciones, sobre todo en las muñecas debido la posición, en la que están sujetas a estrés físico (Torres, 2020).

Trabajar en la computadora implica una posición donde las muñecas están dobladas en un ángulo agudo durante horas. Esto puede producir incomodidad y posiblemente un problema más debilitante llamado síndrome del túnel carpiano (DePaul, 2019). Los síntomas incluyen entumecimiento, hormigueo o dolor que puede presentarse en el pulgar y los dedos, menos el meñique, así como el antebrazo (Ibidem).

\section{Incremento de peso corporal}

El incremento en el peso corporal durante la pandemia ha sido un fenómeno registrado alrededor del mundo en personas que trabajaron de forma remota durante el confinamiento. A este fenómeno se le ha denominado Cuarentena-15, para hacer referencia al aumento promedio de peso expresado en libras, (Wood y Thomas, 2021) y también se le ha llamado Curvas-Covid (Katella, 2020).

En cuestión de género, a nivel mundial más mujeres que hombres reportaron haber tenido un incremento en el peso corporal, mientras que el incremento en número de kilos fue mayor en el caso de los hombres (O'Kane, 2021). Los motivos son diversos, como la disrupción de la rutina y un incremento en el estrés, que han hecho que las personas coman más porque pueden consumir sus alimentos mientras trabajan, situación que no ocurría mientras estaban en una oficina. De hecho, se ha considerado que la pandemia ha creado la tormenta perfecta para aquellas personas que sufren de problemas de sobrepeso y obesidad (Viana en Katella, 2020). Los trabajadores remotos pasan la mayor parte del tiempo sentados y tienen acceso cercano a la comida, por lo que terminan comiendo mucho más que antes. Además, la falta de ejercicio por el confinamiento y la reducción de otras actividades también se relacionan con este fenómeno.

Problemas asociados con la pandemia, como la falta de horas de sueño provocado por el estrés tecnológico, también afectan la cuestión del peso, ya que generan una sensación de hambre (Wood y Thomas, 2021).

Este incremento en el peso no se relaciona ni con la ubicación geográfica ni con comorbilidades. La causa principal es el comfort eating (alimentación reconfortante), seguido por la comodidad de pedir comida a domicilio, o la preparación de más alimentos y postres en casa como una estrategia para matar el tiempo y realizar actividades en familia, así como el incremento en los niveles de estrés (Kelly, 2020).

\section{Síndrome de visión de computadora/ tensión ocular digital}

Otra problemática que se ha incrementado durante el trabajo remoto es la fatiga visual digital. Esta incluye la resequedad y la irritabilidad de los ojos que se agrava al incrementarse las horas frente a la pantalla y al no mantener una distancia adecuada con respecto de la misma (News Center 1, 2020).

Cuando se enfoca la visión en un objeto cercano como una pantalla, los músculos diminutos dentro de los ojos se contraen. La contracción cambia la forma de las lentes dentro de los ojos, enfocando la imagen hacia la retina. Esos pequeños músculos, y otros alrededor de las cuencas de los ojos que mantienen los ojos mirando en la misma dirección, necesitan un descanso (Brown, 2021). Estudios realizados a nivel mundial indican que entre el $22 \%$ y el $38 \%$ de quienes han trabajado de manera remota consideran 
que su visión se ha visto afectada desde el inicio de la pandemia (Ibídem).

Algunos síntomas de este tipo de padecimiento son: visión borrosa, especialmente por la mañana (Leckey, 2021), tener que concentrarse demasiado tiempo en tratar de leer tipografía pequeña en una pantalla iluminada, ojos rojos, resecos, y la sensación de picazón o ardor (Ibídem). Esta situación es relevante debido a que el exceso de resequedad en los ojos los hace más propensos a infecciones (Brown, 2021).

\section{Dolores de cabeza}

Cambios en el ambiente de trabajo y en los horarios laborales han contribuido a una mayor incidencia de dolores de cabeza tensionales y migrañas. La sensibilidad a la luz azul (que emiten las pantallas de los teléfonos celulares y computadoras) es un detonador importante, particularmente ahora que los trabajadores remotos pasan más horas en dispositivos digitales (Upham, 2021). El aumento en los niveles de estrés también contribuye a dolores de cabeza y migraña.

\section{Problemas de sueño}

Informes desarrollados aún antes de la pandemia han sugerido que trabajar desde casa podría estar causando insomnio (Kekatos, 2017). Cuando las personas están acostumbradas a ir a la oficina a trabajar y después regresar a casa a relajarse y cambian esa rutina para integrar ambas actividades en el dominio del hogar, sus ritmos circadianos se confunden, lo que dificulta que la mente determine cuándo es hora de trabajar y de descansar (Kiger, 2020).

Aunado a este fenómeno, un estudio realizado al comienzo de la pandemia reveló tasas muy altas de insomnio que se relacionan con dos grandes factores. Primero, el bajo desapego psicológico que existe con respecto al trabajo en modelos de empleo remoto se relaciona con una calidad de sueño insuficiente por la noche y fatiga por la mañana (Mostafa, 2021). Asimismo, los seres humanos necesitan algo de estimulación y variedad en las actividades cotidianas, cuando la vida se vuelve repetitiva, la falta de dinamismo contribuye a dormir mal. A esta condición se le ha denominado "coronasomnia", haciendo referencia al insomnio producido por la pandemia de coronavirus (UC Davis Health, 2020).

\subsubsection{Problemas de salud mental}

Una de las consideraciones más importante sobre el teletrabajo ha sido la salud mental de los empleados. Las condiciones propias de esta forma de trabajo han conducido al agotamiento, el aislamiento y la ansiedad. Estudios desarrollados por Oracle (2020) sugieren que 2020 ha sido el año más estresante de la historia y que la salud mental de los empleados se ha visto afectada de tal forma que ha impactado también la salud física. La pandemia ha provocado una revalorización a la salud mental debido a un incremento en los niveles de estrés de un porcentaje significativo de teletrabajadores.

\section{Sentimientos de aislamiento o soledad}

Los efectos y las implicaciones del aislamiento en el trabajo remoto no han sido analizados al detalle, sin embargo, la contingencia sanitaria ha puesto de manifiesto la necesidad de su estudio. Muchos empleados, quienes se movieron al empleo remoto, tenían en el lugar de trabajo un espacio para la socialización, dejar dicho entorno y moverse a sus hogares ha tenido un impacto psicológico importante, especialmente en casos en que había otras situaciones preexistentes que pudieran agravarse durante el aislamiento (Zielinska y Kirtzman, 2021).

Cabe destacar que la soledad es una respuesta emocional a la falta de conexión, mientras que el aislamiento está relacionado con el acceso, o la falta de él. Los empleados que se enfrentan al aislamiento en el trabajo carecen de los materiales o la información que necesitan o piensan que se ignoran sus logros o desarrollo (Hickman, 2019). El aislamiento es un problema más técnico que emocional, a diferencia de la soledad, sin embargo, la 
soledad puede contribuir al aislamiento y viceversa.

\section{Estrés}

El estrés derivado de la pandemia puede estar relacionado con múltiples causas, que van desde el miedo al contagio hasta la seguridad laboral, pasando por todo lo que implica el trabajo desde casa, el encierro, la convivencia forzada o el aislamiento (Montaudon-Tomas, Pinto-López, OliveraPérez y Amsler, 2021). Cuando la pandemia llegue a su fin, lo cual eventualmente sucederá, muchas personas habrán vivido todo tipo de tensiones y pérdidas, pérdidas de seres queridos, pérdidas de puestos de trabajo, la quiebra de empresas, etc. De igual manera, para algunas personas, sus matrimonios y otras relaciones sufrirán dificultades producidas por el estrés del auto-aislamiento y los crecientes problemas financieros.

La crisis sanitaria también produjo otros tipos de estrés relacionados con la tecnología, que han sido considerados como enfermedades modernas que se pueden producir a partir de la inhabilidad para el manejo de las nuevas tecnologías, afectando de forma negativa la salud mental (Ingusci, Signore, Giancespro, Manuti, Molino, Russo, Zito y Cortese, 2021). Quienes experimentan este tipo de estrés también suelen ser personas que están expuestos a las tecnologías de la información por periodos prolongados (Bondanini, Giorgi, Ariza-Montes, VegaMuñoz y Andreucci-Annunziata, 2020).

El estrés puede conducir a numerosos problemas de salud, afectando el estado psicológico y fisiológico e incidiendo en los comportamientos y la cognición de las personas (Houtman, Jettinghof y Cedillo, 2007).

\section{Ansiedad}

Un aumento en los niveles de ansiedad también está entre las consecuencias de la pandemia y las condiciones del trabajo remoto. La incertidumbre sobre el futuro y la vuelta a la normalidad hace que las personas se sientan más estresadas, tensas, y hasta agresivas.

El creciente miedo y la ansiedad entre la población mundial se intensifica a partir de las medidas esenciales, pero socialmente disruptivas, como las cuarentenas, los cierres y el distanciamiento social. Esto puede llevar a trastornos psicológicos y psiquiátricos importantes, como estrés postraumático, depresión, ansiedad, pánico y cambios de comportamiento (Sood, 2020).

\section{Burnout}

El síndrome de burnout ha sido definido como la consecuencia emocional generada en un individuo en relación con el trabajo que realiza. Tiene tres características principales: alto desgaste emocional, despersonalización y falta de realización personal (Morales-Cadena, Fonseca-Salazar y Fonseca-Chávez, 2020). Este síndrome se presenta cuando la persona está expuesta constantemente a situaciones laborales que implican un estado de sobrecarga emocional, horarios de trabajo extensos, trabajo físico excesivo, altos niveles de estrés, escasa autonomía, malas relaciones en el entorno laboral, sentimientos de incapacidad para desempeñar las tareas encomendadas, entre otras (Organización Mundial de la Salud, 2019; Martínez-Mejía, Villa-George, Martínez-Guerrero, 2020).

\section{Fatiga}

La fatiga pandémica se ha presentado como una respuesta natural a la prolongada crisis de salud pública y a la desmotivación derivada de las recomendaciones de protección establecidas. Este tipo de cansancio aumenta gradualmente con el tiempo, y las personas que lo padecen se ven afectadas por una variedad de emociones, experiencias y percepciones que generan agotamiento (Organización Mundial de la Salud, 2020). Este tipo de cansancio excesivo está vinculado a la insatisfacción, la incomodidad, la disonancia entre mente y cuerpo, así como un exceso de las demandas laborales (Zerbe, 2020).

\subsection{Riesgos de accidentes}


Los accidentes en el hogar son frecuentes. En diversos países del mundo, el envenenamiento por productos tóxicos, incluyendo inhalación de gas y monóxido de carbono es la causa número uno de muerte accidental en el hogar, seguido por las caídas (Sandorf, 2019). Los tropiezos, resbalones y caídas se han incrementado de manera importante desde que los empleados trabajan de manera remota debido al número de horas que pasan en los hogares y a condiciones particulares como el uso de cables para cargar los dispositivos digitales en superficies que no cuentan con instalaciones eléctricas. Esto se debe a que los hogares no cuentan con los estándares de seguridad de las oficinas o fábricas.

Los empleados remotos suelen tropezarse con objetos como juguetes de los hijos, patinarse por no utilizar zapatos adecuados o por derrames de agua para el café o el té, e incluso caerse caminando mientras envían un mensaje de texto o caerse de las escaleras por correr a atender una llamada telefónica (Previsor Insurance, 2020).

\subsection{Consideraciones adicionales}

Aunado a los problemas de salud derivados del trabajo remoto, existen otras problemáticas ligadas a la pandemia que también deben analizarse. Considerando el alcance actual de la crisis sanitaria, se pueden prever implicaciones de largo plazo en términos socioeconómicos, pero también psicológicos.

Con base en investigaciones sobre las consecuencias sociales y psicológicas de desastres naturales, se estima que, como resultado de la pandemia actual, el 10\% de las personas desarrollarán problemas psicológicos graves, aunque la cifra podría ser mayor (Taylor y Asmundson, 2020). Cabe destacar que, así como existen poblaciones vulnerables ante el COVID-19, también las hay cuando se trata del estrés que genera la expansión del virus y las limitaciones que conlleva.

Personas que viven lejos de sus familias a causa del trabajo o la educación o que simplemente están separados de sus seres queridos tienen un mayor riesgo de desarrollar problemas de salud mental como depresión y ansiedad. Las personas mayores que viven solas están también muy predispuestas (Sood, 2020). Asimismo, la pandemia ha exacerbado los factores de estrés en los sistemas de salud y el burnout de los médicos ya es una epidemia.

En cuanto a las consecuencias de estos niveles de estrés, y tomando en consideración la definición de trauma de acuerdo a los profesionales de la salud mental (acontecimiento estresante que está fuera del alcance de la experiencia humana habitual y que sería muy abrumador para casi cualquier persona ya que implica una percepción de amenaza para la vida o la integridad física), la COVID-19 y la amenaza a la seguridad personal que ha producido cumplen las características de un evento traumático que puede conducir al desarrollo de un trastorno de estrés agudo y hasta un trastorno de estrés postraumático (Restauri y Sheridan, 2020).

$\mathrm{Si}$ a lo anterior se agregan los problemas de salud severos que enfrentarán las personas que padecieron COVID-19, como lo son daños e irregularidades en los pulmones, corazón y riñones $\mathrm{y}$, en los pacientes que estuvieron en cuidados intensivos, dificultades cognitivas y otros problemas mentales (Servick, 2020), la problemática relacionada con la salud física y mental que enfrentarán las sociedades en el futuro será considerable.

\subsection{Las nuevas normas}

A nivel nacional e internacional han comenzado a desarrollarse diversos ordenamientos para prevenir problemas de salud ocupacional relacionados con el trabajo remoto.

La norma mexicana "NOM-035-STPS2018 sobre Factores de Riesgo Psicosocial en el Trabajo, Identificación, Análisis y Prevención" tiene como objetivo sentar las bases para identificar, analizar y prevenir los factores de riesgo psicosocial; así como para promover un entorno organizacional favorable en los centros de trabajo. Los riesgos que se 
consideran son aquellos que pueden provocar trastornos de ansiedad; estrés grave derivado del puesto de trabajo, la jornada laboral o la exposición a acontecimientos traumáticos y/o violencia laboral; y trastornos de los ciclos de sueño (DOF, 2018).

De igual forma, la reforma al Artículo 311 de la Ley Federal del Trabajo en materia de Teletrabajo o Home office establece los derechos básicos de las personas que laboran bajo este esquema, donde se incluye: recibir el equipo necesario para realizar las actividades laborales, recibir salario/pago en la forma y fechas estipuladas, percibir los costos derivados del trabajo como telecomunicación y electricidad, trato correcto de la información y los datos utilizados, desconexión al término de la jornada laboral, derechos en cuanto a seguridad social, capacitación y asesoría y perspectiva de género para asegurar la conciliación de la vida familiar y laboral (DOF, 2021).

\subsection{Recomendaciones}

Los problemas de salud presentados han sido identificados por diversas empresas, instituciones de salud y organismos, quienes se han dado a la tarea de emitir una serie de recomendaciones para limitar su aparición, así como para reducir sus efectos y su impacto. A continuación, se presenta una compilación de las recomendaciones más relevantes.

\subsubsection{Protección a la salud física}

\section{Tech Neck o cuello tecnológico}

Mantener una buena postura es fundamental, por lo que se recomienda repensar la estación de trabajo en casa, revisar el tipo de silla y la altura de la silla y la mesa (Healthline, 2020a). Usar una silla ergonómica es ideal, por lo que se sugiere utilizar una silla que se recline y reclinarse ligeramente hacia atrás en 25 a $30^{\circ}$ (Hansraj, 2014). Si no se cuenta con una silla ergonómica, se pueden utilizar toallas dobladas o cojines para soportar tanto el cuello como la espalda (Chandler, 2020).
Estirarse de manera frecuente también puede ayudar; se recomienda levantarse de la silla al menos cada media hora y caminar un poco (Hansraj, 2014) y realizar ejercicio de forma periódica.

\section{Dolor en la espalda baja}

Lo ideal es recrear el espacio de trabajo de la oficina, llevando, por ejemplo, la silla ergonómica a la casa (Moretti et al., 2020), esto se puede traducir también a las rutinas, recreando los horarios de trabajo para no pasar más tiempo de la jornada laboral sentados frente a la pantalla.

Se debe evitar trabajar desde la cama, a menos que se cuente con una mesa especial para tal propósito (Hedge, 2020). Se recomienda no trabajar en sillones o superficies suaves que no ofrezcan soporte a la espalda (Chandler, 2020). Se puede colocar un descansa pies, caja o algún otro objeto para soporte de los pies (Ibidem) cuando se trabaja en una mesa. Al igual que con los problemas de cuello, realizar ejercicio de manera periódica, así como estiramientos, puede ayudar a prevenir este tipo de padecimientos.

\section{Problemas en articulaciones, síndrome del túnel carpiano, tendinitis}

Se sugiere evitar usar cualquier tipo de acojinamiento de soporte para la muñeca, ya que ello agrega presión a los tendones y los nervios (Hedge, 2020). Asimismo, realizar estiramientos de mano y dedos, al igual que rotaciones (Hand \& Wrist Center, 2020), puede ayudar a prevenir o disminuir el dolor en las articulaciones. DePaul (2019) recomienda ajustar la altura del teclado para reducir la flexión necesaria para escribir, o invertir en un teclado ergonómico que elimine los ángulos severos por completo.

\section{Incremento en el peso corporal o Cuarentena-15}

Registrar los alimentos consumidos a lo largo del día y reducir la ingesta de bebidas azucaradas (Healthcare, 2021) han sido consideradas como buenas estrategias para evitar subir de peso. Hacer ejercicio regularmente y alimentarse de manera 
saludable es importante. También se recomienda atender problemas de salud mental asociados, como el caso de la ansiedad y el aislamiento y tratar de entender cuál es el origen de la necesidad de comer. Buscar la socialización con otros, aun de manera virtual puede servir para canalizar las emociones hacia acciones más positivas (Kelly, 2020).

\section{Síndrome de visión de computadora/ tensión ocular digital}

Reducir el brillo de las pantallas, descansar la vista cada determinado tiempo e hidratar los ojos frecuentemente (Forster, 2020) son buenas prácticas para evitar la tensión ocular relacionada con el trabajo remoto. En este sentido, es importante parpadear de manera frecuente (Greenstein, 2020) a fin de evitar que se resequen los ojos. Si no hay humedad en el ojo, la córnea se reseca y la visión se vuelve borrosa (Brown, 2021). También se puede colocar un humidificador en la habitación donde se trabaja (Vision Health, 2020).

Se recomienda relajar los músculos oculares, utilizando la regla 20/20/20: cada veinte minutos tomar una pausa de veinte segundos para cerrar los ojos y para mirar hacia otro lugar a 20 pies de distancia (aproximadamente 6.5 metros) (Brown, 2021), así como mantener una distancia adecuada con respecto de la pantalla. Lo mejor es colocar la pantalla de la computadora a una distancia aproximada de un brazo extendido (Chandler, 2020).

Se pueden utilizar lentes que filtran la luz azul de las pantallas (Leckey, 2021) y ampliar el tamaño del texto para evitar forzar la vista (Brown, 2021). No es recomendable trabajar con la luz natural de una ventana de frente ni en la espalda, ya que ello afecta la visión. Lo mejor es colocarse con una ventana de costado, lo cual reducirá la exposición directa a la fuente de luz y reducirá los reflejos y destellos dañinos (Hedge, 2020). Se sugiere utilizar cortinas para evitar los reflejos y reducir el riesgo de cansancio ocular (Chandler, 2020).

\section{Dolores de cabeza y migrañas}

Mantenerse hidratado, controlar la ingesta de cafeína y mantenerla lo más regular posible, así como cuidar la dieta limitando la cantidad de azúcares pueden ayudar a prevenir el dolor de cabeza. Las recomendaciones para evitar o disminuir la tensión ocular digital también son aplicables a los dolores de cabeza y migrañas, ya que los síntomas pueden estar relacionados (McCallum, 2020).

\section{Problemas de sueño}

Tratar de acostarse todos los días a la misma hora para generar una rutina es crucial. Aquellos que tienen problemas para dormir deben establecer un horario regular que coincida con sus relojes internos, desarrollar una rutina relajante antes de dormir y reducir la ingesta de luz (especialmente de dispositivos electrónicos) durante una o dos horas antes de acostarse. Las siestas diurnas, la cafeína y el alcohol también pueden afectar el sueño (Kiger, 2020), por lo que evitarlos es fundamental para disminuir los problemas de sueño.

\subsubsection{Protección de la salud mental}

\section{Sentimientos de aislamiento}

Gallup recomienda promover conversaciones frecuentes y continuas, con un lenguaje específico para los trabajadores remotos (Hickman, 2019). La mejor estrategia es crear oportunidades para conexiones significativas, como hacer presentaciones formales a los colegas y vincular al empleado con otros para ciertos proyectos. Los chats grupales en línea también permiten a los equipos mantener una comunicación continua (Ibidem).

\section{Estrés}

Desarrollar respuestas saludables al estrés es una de las mejores estrategias. El ejercicio puede ser de gran ayuda, los expertos recomiendan hacer yoga, pero cualquier forma de actividad física es beneficiosa. Dormir lo suficiente también es importante para el manejo eficaz del estrés, por lo que desarrollar hábitos de sueño saludables limitando el consumo de cafeína al final del día y 
minimizando las actividades estimulantes por la noche es esencial.

Establecer límites entre el trabajo y la vida personal también puede ayudar a disminuir el estrés, al igual que tomarse tiempo para recargar y desconectarse del trabajo (Abrahams, 2019). Algunas empresas han desarrollado aplicaciones digitales útiles como herramientas de telemedicina por medio de teléfonos inteligentes para que sus empleados a distancia puedan controlar sus niveles de estrés y estos puedan ser monitoreados por servicios de salud (Gleason, 2021).

\section{Ansiedad}

La cafeína es inductora de ansiedad y el alcohol cambia los niveles de serotonina y otros neurotransmisores en el cerebro, por lo que evitarlos es fundamental para las personas que sufren de ansiedad (Healthline, 2020). De igual forma, la ansiedad puede derivarse de una acumulación de adrenalina, en estos casos, el ejercicio puede ayudar a usar esa adrenalina adicional. No beber suficiente agua puede empeorar los síntomas de ansiedad ya que la deshidratación puede causar palpitaciones del corazón que llevan a sentimientos de pánico y ataques de ansiedad (Healthline, 2020b).

\section{Síndrome de burnout}

Es esencial reponer la energía física y emocional, para ello se deben priorizar los buenos hábitos de sueño, la nutrición, el ejercicio, los vínculos sociales y otras prácticas que promueven el bienestar. Encontrar puntos de equilibrio es crucial, si se pasa el día en un entorno agitado y ruidoso, un momento de tranquilidad a solas puede ser favorecedor (Blaschka, 2020).

Se recomienda observar de cerca los pensamientos y emociones en relación con el trabajo, preguntándose qué aspectos de la situación están realmente establecidos y cuáles pueden cambiar, esto puede ayudar a cambiar de perspectiva y disminuir el impacto negativo del estrés relacionado con los aspectos inflexibles (Valcour, 2016).

\section{Fatiga}

Algunos psicólogos sugieren que la fatiga derivada del trabajo remoto puede provenir de la necesidad de mayor concentración, sobre todo cuando se realizan reuniones virtuales donde procesar constantemente la comunicación no verbal de otros asistentes implica un gran esfuerzo. Se recomienda que, en la medida de lo posible, las reuniones sean solo de audio para evitar sobrecargas sensoriales (James, 2021). Asimismo, acortar las reuniones de una hora a 45-50 minutos permite a los asistentes tener descansos rápidos entre reuniones. $\mathrm{Al}$ tomar estos descansos de la pantalla es importante no utilizar ese tiempo para revisar otros dispositivos móviles (Ibidem).

\subsubsection{Reducción de accidentes de trabajo}

Mantener los espacios de trabajo ordenados y los cables eléctricos en buen estado, no pasar los cables por debajo de los tapetes ni dejarlos en lugares donde estorben el paso, desconectar todos los dispositivos y cables que no se encuentren en uso, así como no conectar muchos cables en el mismo contacto o extensión para evitar sobrecargas eléctricas son algunas recomendaciones para evitar accidentes trabajando desde casa.

De igual manera, es prudente contar con una alarma anti humo y para monóxido de carbono, un extintor para fuegos eléctricos y un kit de primeros auxilios. Almacenar de forma adecuada los productos peligrosos es de vital importancia. Se sugiere evitar los tapetes sueltos que pudieran provocar tropiezos y sostenerse del pasamanos y no cargar demasiadas cosas, como computadora y materiales, mientras se baja la escalera para dejar siempre una mano libre (Previsor Insurance, 2020).

Para los trabajadores remotos, evitar bebidas en contenedores abiertos cerca de los contactos de luz o las extensiones eléctricas (Ibidem), al igual que no colocar las computadoras o dispositivos cerca de una fuente de calor y verificar que no se sobre calienten son algunas estrategias que pueden ayudar a evitar percances. 


\section{CONCLUSIONES}

Actualmente, el trabajo remoto ya no es únicamente para empleados altamente calificados con tareas y esquemas de trabajo relativamente autónomos, sino que su uso se ha expandido hasta llegar a competir con la vida privada y el tiempo libre. Todas las empresas, organizaciones e instituciones se verán cada vez más afectadas por las distintas aplicaciones de las Tecnologías de la Información y la Comunicación.

Con la crisis de salud ocasionada por la pandemia de COVID-19, el número de personas que practican teletrabajo incrementaron sustancialmente, y numerosas organizaciones han contemplado conservar esquemas de trabajo remoto o trabajo híbrido aun cuando las actividades vuelvan a la normalidad.

Esta situación ha probado que el teletrabajo es una solución en tiempos complejos y que, en muchos sectores, se trata de un método sostenible que además reduce costos relacionados con la presencia de los empleados en un espacio laboral físico. Sin embargo, la transición forzada de la rutina laboral al ámbito digital y las condiciones generales del empleo relacionadas con ella resultaron en una serie de problemáticas que han incidido en la salud y el bienestar de los trabajadores remotos, que deberán atenderse si se pretende conservar o ampliar este tipo de esquemas de trabajo.

Las innovaciones tecnológicas constantes y su impacto en las organizaciones permiten asegurar que el futuro de los negocios, el trabajo y la economía será digital. En este contexto, es fundamental profundizar la investigación sobre el impacto físico y psicológico del trabajo remoto, con el objetivo de identificar, prevenir y gestionar los riesgos correspondientes. El establecimiento de medidas para asegurar el bienestar físico y mental de los empleados remotos es crucial, particularmente si se considera la proliferación inminente del teletrabajo.
Una recomendación de interés es el desarrollo de círculos de salud. Se trata de grupos de empleados o círculos de empleados que han sido utilizados en diversos campos. Es posible que los más conocidos sean los círculos de calidad. Un círculo de calidad es un grupo de empleados que se reúne periódicamente para solucionar problemas que afectan a su área de trabajo (Lawler y Morhman, 1985). Generalmente se trata de 6 a 12 personas de la misma área quienes reciben capacitación en resolución de problemas, procesos grupales y asuntos de calidad.

Por su parte, los círculos de salud o círculos saludables han sido utilizados para empoderar a los empleados y fomentar su participación en la salud organizacional. Los empleados son considerados expertos para identificar las condiciones de trabajo que generan problemas y estrés y por lo tanto son la fuente más adecuada para identificar las mejores estrategias de intervención (MunnGiddings, Hart y Ramos., 2005; Aust y Ducki, 2004; Linos y Kirch, 2008). Desarrollar este tipo de grupos permitirá a los empleados monitorear los riesgos y sus propios problemas de salud, así como los de sus compañeros aun a la distancia y promover acciones que reduzcan la sensación de aislamiento, favoreciendo la salud ocupacional. Estos grupos deberán reportar el estado de salud y bienestar de los trabajadores remotos a la autoridad competente, como es el caso de recursos humanos o las áreas similares. Dichos reportes permitirán generar políticas y estrategias de acuerdo con las necesidades de quienes colaboran a la distancia, más allá del simple cumplimiento con los preceptos legales, favoreciendo una verdadera cultura del cuidado.

Existen numerosas otras prácticas que se pueden implementar para mejorar la salud ocupacional en la modalidad remota del empleo. El propósito central del presente artículo consistió en describir los distintos problemas de salud asociados al teletrabajo, así como recomendaciones para el desarrollo 
de investigaciones empíricas y otros estudios que impulsen el desarrollo de medidas para

promover el bienestar en el trabajo remoto.

\section{REFERENCIAS}

Abrahams, Z. (2019). The future of remote work: When it's done right, telework can improve employee productivity, creativity and morale, psychologists' research finds. American Psychological Association. https://www.apa.org/monitor/2019/10/cover-remote-work

Allen, T. D., Cho, E., y Meier, L. L. (2014). Work-family boundary dynamics. Annual Revue Organizational Psychology \& Organizational Behavior, 1 (1), 99-121.

Aust, B., y Ducki, A. (2004). Comprehensive health promotion interventions at the workplace: experiences with health circles in Germany. Journal of occupational health psychology, 9(3), 258.

Berg, J., Bonnet, F. y Soares, J. (2020). Working from home: estimating the worldwide potential. Vox CEPR Policy Portal. https://voxeu.org/article/working-home-estimating-worldwidepotential

Blaschka, A. (2020). This Is The Best Way To Avoid COVID Burnout. Forbes. https://www.forbes.com/sites/amyblaschka/2020/07/30/this-is-the-best-way-to-avoidcovid-burnout/?sh=35e055a0509d

Bondanini, G., Giorgi, G., Ariza-Montes, A., Vega-Muñoz, A., \& Andreucci-Annunziata, P. (2020). Technostress dark side of technology in the workplace: a scientometric analysis. International Journal of Environmental Research and Public Health, 17(21), 8013.

Brown, D. (2021). Covid-19: Five ways to avoid lockdown eye strain. BBC News. https://www.bbc.com/news/uk-55933168

Cameron, D. (2021). History of Workplace Health and Safety. Staysafe. https://staysafeapp.com/blog/2020/08/21/history-workplace-health-and-safety/

Chandler, C. (2020). Working from home occupational health and safety implications. https://www.lexology.com/library/detail.aspx?g=9fbe1053-b59e-4fe9-b6c1$5 \mathrm{e} 45 \mathrm{~d} 8489042$

DePaul, K. (2019). Remote Work Health Risks: What You Need to Know. Remote Co. https://remote.co/remote-work-health-risks-what-need-

know/\#: :text=Carpal\%20Tunnel\%20Syndrome,problem\%20called\%20Carpal\%20Tun nel\%20Syndrome

Di Donato, M., Iles, R., Buchbinder, R., Xia, T., y Collie, A. (2021). Prevalence, Predictors and Wage Replacement Duration Associated with Diagnostic Imaging in Australian Workers with Accepted Claims for Low Back Pain: A Retrospective Cohort Study. Journal of Occupational Rehabilitation, 1-9.

DOF Diario Oficial de la Federación (2018). Norma Oficial Mexicana NOM-035-STPS-2018, Factores de riesgo psicosocial en el trabajo-Identificación, análisis y prevención. https://www.dof.gob.mx/nota_detalle.php?codigo=5541828\&fecha=23/10/2018)

DOF Diario Oficial de la Federación (2021). Decreto por el que se reforma el artículo 311. https://dof.gob.mx/nota_detalle.php?codigo $=5609683 \&$ fecha $=11 / 01 / 2021 \#: \sim:$ text=Art\% C3\%ADculo\%20330\%2DC.,sus\%20labores\%20bajo\%20esta\%20modali dad.

EHS Insight resources (2019). A Brief History of Occupational Health and Safety. https://www.ehsinsight.com/blog/a-brief-history-of-occupational-health-andsafety\#: :text=In\%20the\%20United\%20States $\% 2 \mathrm{C} \% 20$ occupational,of $\% 20$ their\%20job $\% 20$ or\%20industry.

Foro Económico Mundial. (2020). The Future of Jobs Report 2020. World Economic Forum, Geneva, Switzerland.https://www.weforum.org/reports/the-future-of-jobs-report-2020

Forster, V. (2020). Are Your Eyes Hurting During The Coronavirus Pandemic? You May Have "Computer Vision Syndrome" Forbes https://www.forbes.com/sites/victoriaforster/2020/06/01/are-your-eyes-hurting-during- 
the--coronavirus-pandemic-you-may-have-computer-vision-

syndrome/?sh=32dcb8326cf9

Gleason, A. M. (2021). Remote Monitoring of a Work-From-Home Employee to Identify Stress: A

Case Report. Workplace Health \& Safety, https://journals.sagepub.com/doi/abs/10.1177/2165079921997322

Greenstein, M. (2020). Tips for eye health while working from home. https://www.kshb.com/news/coronavirus/tips-for-eye-health-while-working-from-home

Hand \& Wrist Center (2020). 4 Hand-Wrist Stretches for Remote Workers During COVID19https://northfloridahandandwrist.com/4-hand-wrist-stretches-for-remote-workersduring-covid-19/

Hansraj, K. K. (2014). Assessment of Stresses in the Cervical Spine Caused by Posture and Position of the Head; Surg Technol Int.25:277 -279.

Healthcare (2021). Pandemic Weight Gain — It's a Thing. https://www.muhealth.org/ourstories/pandemic-weight-gain-its-thing

Hedge, A. (2020). Getting Back Pain While Working From Home? An Ergonomics Expert Offers Advice. Time magazine. https://time.com/5821252/back-pain-work-from-home-tips/

Healthline (2020a). 4 Simple Tips to Avoid Developing 'Tech Neck' During COVID-19. https://www.healthline.com/health-news/4-simple-tips-to-avoid-developing-tech-neckduring-covid-19\#How-to-treat-tech-neck

Healthline. (2020b). 12 Ways to Calm Your Anxiety. https://www.healthline.com/health/how-tocalm-anxiety

Hickman, A. (2019). How to Manage the Loneliness and Isolation of Remote Workers. Gallup. https://www.gallup.com/workplace/268076/manage-loneliness-isolation-remoteworkers.aspx

Houtman, Irene, Jettinghof, Karin, Cedillo, Leonor \& the World Health Organization. Occupational and Environmental Health Team. (2007). Raising awareness of stress at work in developing countries : advice to employers and worker representatives. World Health Organization. https://apps.who.int/iris/handle/10665/42956

Ingusci, E., Signore, F., Giancaspro, M. L., Manuti, A., Molino, M., Russo, V., ... \& Cortese, C. G. (2021). Workload, Techno Overload, and Behavioral Stress During COVID-19 Emergency: The Role of Job Crafting in Remote Workers. Frontiers in Psychology, 12.

James, J. (2021). Remote work exhaustion: 13 tips to reduce fatigue. The Enterprises Project. https://enterprisersproject.com/article/2021/1/remote-work-13-tips-reduce-fatigue

Katella, K. (2020). Quarantine 15? What to Do About Weight Gain During the Pandemic. Yale Medicine. https://www.yalemedicine.org/news/quarantine-15-weight-gain-pandemic

Kekatos, M. (2017). Work at home? Watch out! You're more likely to suffer insomnia, stress and depression. Daily Mail. https://www.dailymail.co.uk/health/article-4227786/Workhome-likely-suffer-insomnia.html

Kelly, J. (2020). Q\&A: WHY PEOPLE ARE ‘STRESS EATING' DURING THE PANDEMIC, AND HOW TO STOPhttps://news.virginia.edu/content/qa-why-people-are-stress-eatingduring-pandemic-and-how-stop

Kiger, P. J. (2020). Pandemic Is Turning Some Older Telecommuters Into Insomniacs. AARP. https://www.aarp.org/work/working-at-50-plus/info-2020/work-from-homeinsomnia.html

Lawler E. E., y Mohrman, S. A. (1985). Quality circles after the fad. Harvard Business Review, 63(1), 65-71.

Leckey, R. (2021). A year of screens: How more remote work \& virtual learning affects our eye health https://www.wnep.com/article/entertainment/television/leckey-live/computerscreens-remote-work-virtual-learning-eye-health-pandemic/523-9652813e-1242-41bfbf05-0a54f6a7c397 
Linos, A., y Kirch, W. (Eds.). (2008). Promoting health for working women. Springer.

Martínez-Mejía, E., Villa- George, F. I., y Martínez-Guerrero, J. I. (2020). Prevalence of Burnout Syndrome in Mexican Employees in Mexico City. Journal of Basic and Applied Psychology Research, 2(3), 23-33.

Maruyama, T., Hopkinson, P. G., y James, P. W. (2009). A multivariate analysis of work- life balance outcomes from a large-scale telework programme. New Technology, Work and Employment, 24(1), 76-88.

McCallum, K. (2020). Is Excess Screen Time Causing Your Headaches?. Houston Methodist. https://www.houstonmethodist.org/blog/articles/2020/apr/is-extra-screen-time-causingyour-headaches/

Miller, L. (2020). How bad is working from home making your tech neck?, EWIWORKS. https://www.ewiworks.com/blog/bad-text-neck/

Montaudon-Tomas, C., Pinto-López, C.M., Olivera-Pérez, E. y Amsler, A. (2021). Informe: El Estado del Trabajo Remoto en México durante la Pandemia de COVID-19. Observatorio de Competitividad y Nuevas Formas de Trabajo. Puebla: UPAEP Universidad.

Morales-Cadena, G. M., Fonseca-Salazar, N. F., y Fonseca-Chávez, M. G. (2020). Prevalence of professional burnout syndrome in postgraduate residents of surgical and non-surgical specialties. Anales de Otorrinolaringología Mexicana, 65 (2), 64-70.

Moretti, A., Menna, F., Aulicino, M., Paoletta, M., Liguori, S., y Iolascon, G. (2020). Characterization of Home Working Population during COVID-19 Emergency: A CrossSectional Analysis. International Journal of Environmental Research and Public Health, 17(17), 6284.

Mostafa, B. A. (2021). The Effect of Remote Working on Employees Wellbeing and Work-Life Integration during Pandemic in Egypt. International Business Research, 14(3), 41-52.

Munn-Giddings, C., Hart, C., y Ramos, S. (2005). A participatory approach to the promotion of well-being in the workplace: Lessons from empirical research. International Review of Psychiatry, 17(5), 409-417.

News Center 1. (2020). How to alleviate digital eye strain while working from home. https://www.newscenter1.tv/how-to-alleviate-digital-eye-strain-while-working-fromhome/

O'Kane, C. (2021). Most Americans say the pandemic has been bad for their weight. CBS News. https://www.cbsnews.com/news/covid-weight-gain-30-pounds-pandemic/

Organización Internacional del Trabajo. (2017). The future of Work. International Labor Organization. Geneva. Disponible en: https://www.ilo.org/global/topics/future-ofwork/langen/index.htm

Organización Internacional del Trabajo. (2020). Defining and measuring remote work, telework, work at home and home based work. https://www.ilo.org/global/statistics-anddatabases/publications/WCMS_747075/lang--en/index.htm

Organización Mundial de la Salud. (2021). Occupational health. https://www.who.int/healthtopics/occupational-health

Organización Mundial de la Salud. (2019). Burn-out an "occupational phenomenon": International Classification of Diseases. https://www.who.int/news/item/28-05-2019-burn-out-anoccupational-phenomenon-international-classification-of-diseases

Oracle. (2020). As Uncertainty Remains, Anxiety and Stress Reach a Tipping Point at Work: Artificial intelligence fills the gaps in workplace mental health support. https://www.oracle.com/a/ocom/docs/oracle-hcm-ai-at-work.pdf

Porter, R. (2021). Is COVID-19 Causing Carpal Tunnel?. Korn Ferry. https://www.kornferry.com/insights/this-week-in-leadership/is-covid-19-causing-carpaltunnel

Potratz, A. (2020). Managing remote workers during the COVID-19 Pandemi,. https://ohsonline.com/Articles/2020/08/09/Managing-Remote-Workers-During-the- 
COVID-19-Pandemic.aspx?Page $=7$

Previsor Insurance. (2020). Tips to Avoid Work-Related Injuries While Working from Home. https://previsorinsurance.com/blog/tips-to-avoid-work-related-injuries-while-workingfrom-home/

Restauri, N. y Sheridan, A. (2020). Burnout and Posttraumatic Stress Disorder in the Coronavirus Disease 2019 (COVID-19) Pandemic: Intersection, Impact, and Interventions. Journal of the American College of Radiology. Disponible en: https://www.jacr.org/article/S15461440(20)30546-9/fulltext

Sandorf, B. (2019). Home Accident Statistics: Is Your Home as Safe as You Think? https://www.asecurelife.com/home-accident-statistics/

Servick, K. (2020). For survivors of severe COVID-19, beating the virus is just the beginning. Science Magazine. Disponible en: https://www.sciencemag.org/news/2020/04/survivorssevere-covid-19-beating-virus-just-beginning

Sood, S. (2020). Psychological effects of the Coronavirus disease-2019 pandemic. RHiME. 7. 236.

Tadesse, T. y Admassu, M. (2006). Occupational Health and Safety. Carter Center. https://www.cartercenter.org/resources/pdfs/health/ephti/library/lecture_notes/env_occu pational_health_students/ln_occ_health_safety_final.pdf

Taylor, S. y Asmundson, G. (2020). Life in a post-pandemic world: What to expect of anxietyrelated conditions and their treatment. Journal of anxiety disorders. https://www.ncbi.nlm.nih.gov/pmc/articles/PMC7252157/

The Center of Occupational Safety. (2020). Mobile work and remote work. Finlandia, https://ttk.fi/en/well-

being_at_work_and_occupational_health_and_safety/the_basics_for_occupational_safet y_and_health/work_environment/mobile_work_and_remote_work

Torres, M. (2020). This Is What Happens To Your Body When You Work From Home. Huffpost. https://www.huffpost.com/entry/what-happens-body-work-fromhome_1_5e84bfb5c5b6a1bb765185df

UC Davis Health. (2020). COVID-19 is wrecking our sleep with coronasomnia - tips to fight back. https://health.ucdavis.edu/health-news/newsroom/covid-19-is-wrecking-our-sleep-withcoronasomnia--tips-to-fight-back/2020/09\#: :text=And\%20a\%20report\%20from\%20the,a\%20surprise\%20to\%20no\%20 one.

UNUM. (2020). Remote working and the risks to physical health. https://www.unum.co.uk/resources/remote-working-and-the-risks-to-physical-health

Upham, B. (2021). The Link Between Migraine Headache and Neck Pain. Everyday Health. https://www.everydayhealth.com/migraine/the-link-between-migraine-headache-andneck-pain/

Valcour, M. (2016). Beating Burnout. Harvard Business Review. https://hbr.org/2016/11/beatingburnout

Vision Health (2020). Tips to Reduce Eye Strain When Working From Home. https://www.deltadentalia.com/a-healthy-life/vision-health/tips-to-reduce-eye-strainwhen-working-from-home/

Web of Science (2021). "Remote work" and "occupational health" https://apps-webofknowledgecom.erevistas.ugto.mx/full_record.do?product=WOS\&search_mode=GeneralSearch\&qid=1\& $\mathrm{SID}=6 \mathrm{ASVjb5ZFaxr} 4 \mathrm{SpNINB} \&$ page $=1 \& \mathrm{doc}=4$

Wood, A. y Thomas, N. (2021). If you've gained weight during the pandemic, you're not alone. Here's what to do about it. CNN. https://www.cnn.com/2021/03/24/health/weight-gainduring-covid-wellness/index.html

Zerbe, K. J. (2020). Pandemic fatigue: Facing the body's inexorable demands in the time of Covid- 
19. Journal of the American Psychoanalytic Association, 68(3), 475-478.

Zielinska, M. Y Kirtzman, F. (2021). The Mental Health Toll of Working in Isolation. Korn Ferry. https://www.kornferry.com/insights/this-week-in-leadership/mental-health-remotework-coronavirus 